\title{
A Novel Approach to the Treatment of Pembrolizumab-induced Psoriasis Exacerbation: A Case Report
}

Elio P. Monsour ${ }^{1}$, Joshua Pothen ${ }^{2}$, Rama Balaraman ${ }^{3}$

1. Internal Medicine, University of Central Florida / Ocala Regional Medical Center, Ocala, USA 2. Internal Medicine, General Medical Education, Ocala Regional Medical Center, Ocala, USA 3. Hematology and Oncology, Florida Cancer Affiliates / Ocala Oncology, Ocala, USA

Corresponding author: Elio P. Monsour, eliomonsour@gmail.com

\begin{abstract}
While immune checkpoint inhibitors have been groundbreaking for cancer treatment, there are many reported cases of patients undergoing immunotherapy who have discontinued or temporarily interrupted treatment due to the development of autoimmune-related adverse effects. Here, we present a 63-year-old female with a history of psoriasis (in spontaneous remission) and newly diagnosed poorly differentiated lung adenocarcinoma (pTXN3M1a) who experienced a severe flare-up of her psoriasis three months after initiating single-agent pembrolizumab. The patient was initially treated with topical clobetasol propionate ointment, however, due to minimal response to this regimen, the patient was commenced on secukinumab; an IL-17 inhibitor. To our knowledge, this is the first case of the successful use of secukinumab for the treatment of immunotherapy-induced psoriasis. More importantly, immunotherapy with pembrolizumab was continued successfully with the co-administration of secukinumab without complications or the recurrence of non-small cell lung cancer (NSCLC).
\end{abstract}

Categories: Dermatology, Internal Medicine, Oncology

Keywords: immunotherapy in lung cancer, psoriasis, pembrolizumab, oncology, dermatology, pd-11 inhibitor

\section{Introduction}

Immune checkpoint inhibitors have been a breakthrough for cancer therapy in recent years, especially for the treatment of metastatic non-small cell lung cancer (NSCLC). Pembrolizumab is immunotherapy that works by blocking the interaction between the programmed death-ligand 1 (PD-L1) receptor on T-cells with the PD-L1 and PD-L2 ligands on tumor cells [1]. Consequently, this promotes T-cell reactivation and restored immune response, rather than T-cell deactivation. However, immunotherapy is associated with increased immune-related adverse effects. Early discontinuation of immunotherapy may pose a risk to treatment outcomes, and, thus, recognition and management of its underlying side effects are crucial. We report an unusual case of pembrolizumab-induced psoriasis exacerbation treated effectively with

Received 09/19/2019

Review began 09/25/2019 Review ended 09/30/2019 Published 10/02/2019

\section{() Copyright 2019}

Monsour et al. This is an open access article distributed under the terms of the Creative Commons Attribution License CC-BY 3.0., which permits unrestricted use, distribution, and reproduction in any medium, provided the original author and source are credited. secukinumab.

\section{Case Presentation}

A 63-year-old female patient with a history of hypothyroidism, gastroesophageal reflux disease (GERD), hypertension, and psoriasis initially presented to the clinic with complaints of right hip pain. Magnetic resonance imaging (MRI) of the right hip revealed edema in the trochanteric bursa and a mass measuring 3.6 $\mathrm{x} 1.8 \mathrm{~cm}$ in size. Two adjacent nodules in the pelvis concerning for adenopathy were also discovered.

Subsequent whole-body positron emission tomography-computed tomography (PET/CT) scan demonstrated hypermetabolic adenopathy in the neck, chest, and pelvis. Lymph node biopsy of the pelvis established the histological diagnosis of poorly differentiated lung adenocarcinoma, clinically staged as TXN3M1a NSCLC (stage IVb). Genomic molecular analysis showed programmed death-ligand 1 (PD-L1) positivity with >50\% expression without known estimated glomerular filtration rate (EGFR) or ALK genomic tumor aberrations.

Prior to the initiation of immunotherapy with pembrolizumab $200 \mathrm{mg}$ intravenous (IV) every three weeks, the patient had no active psoriatic lesions and had been in spontaneous remission of her psoriasis for the past couple of years. However, three months following immunotherapy, the patient developed diffuse dermatitis on her left ventral proximal forearm, anterior proximal thigh, arms, legs, and trunk. The patient was referred to dermatology, and a shave biopsy was performed. Pathology showed severe plaque psoriasis, most probably induced by pembrolizumab (Figure 1 ). She was started on $0.05 \%$ clobetasol propionate cream but failed to respond to treatment after four weeks. Computed tomography (CT) of the chest, abdomen, and pelvis showed no findings of recurrence or metastatic disease in the chest. Following a negative hepatitis panel and interferon-gamma release assay, the patient was commenced on secukinumab and, gradually, the patient's psoriasis was ameliorated. Given the clinical response to secukinumab for psoriasis, pembrolizumab was continued without further complications (Figure 2). 


\section{Cureus}



FIGURE 1: Histopathology of skin lesions using hematoxylin and eosin staining (approximate magnification 200x) consistent with psoriasis

Adapted from Galluzo et al. [2].
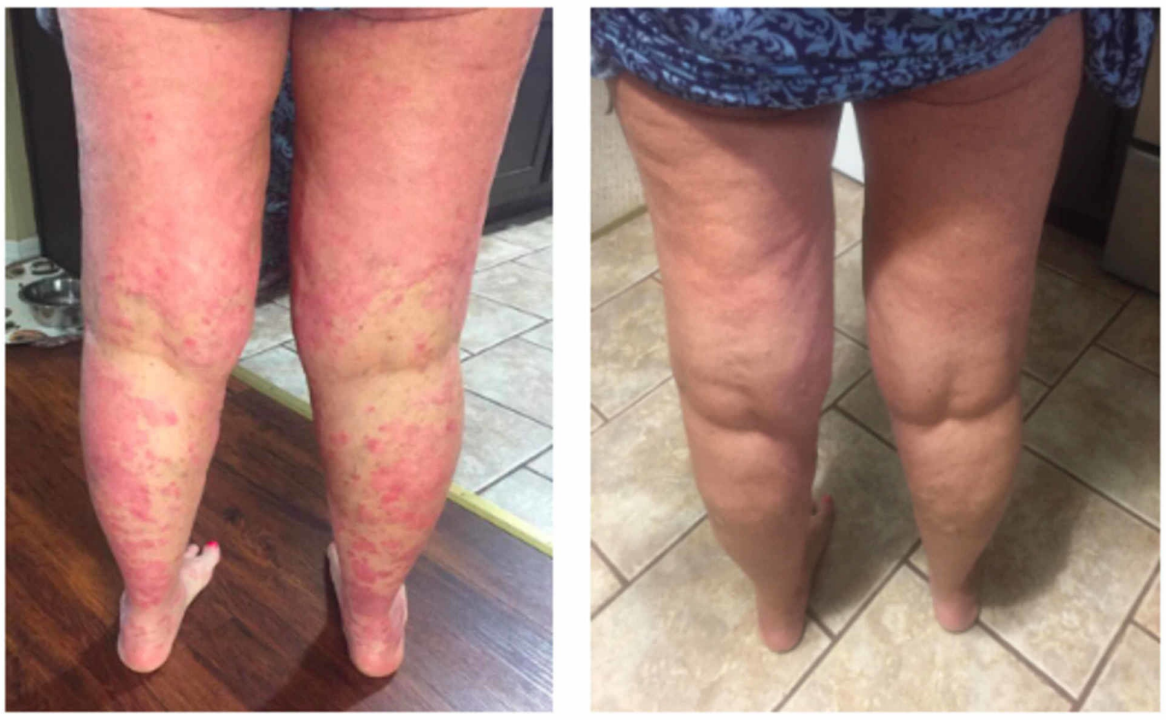

FIGURE 2: Before and after photos following treatment with secukinumab

\section{Discussion}

This case illustrates plaque psoriasis exacerbation following treatment with pembrolizumab, successfully treated with secukinumab. Other etiologies of psoriasis flare-up were ruled out, including but not limited to infections, alcohol intake, skin injury, or use of other medications associated with worsening of psoriasis.

The patient's quality of life was severely affected due to her extensive psoriatic lesions, even though she had no evidence of metastatic disease or recurrence of NSCLC. The etiology of psoriasis and other autoimmune 
Often, treatment of these autoimmune conditions requires the use of immunosuppression therapy and interruption or complete discontinuation of immunotherapy, which may all pose cancer treatment risk outcomes. Secukinumab works by selectively targeting IL-17A and blocks its interaction with the IL-17 receptor, thus inhibiting downstream pro-inflammatory cytokine production (Figure 3) [4]. A review of published literature via PubMed until July 2019 has demonstrated approximately 34 cases of psoriasis due to anti-PD-1/PD-L1 therapy [5]; however, to our knowledge, this is the first case of the successful use of secukinumab for the treatment of immunotherapy-induced psoriasis. More importantly, immunotherapy with pembrolizumab was continued successfully with the co-administration of secukinumab without complications or recurrence of NSCLC.



\section{Conclusions}

We report an interesting case of a patient with PD-L1 positive advanced non-small cell lung cancer who developed severe exacerbation of her psoriasis secondary to treatment with pembrolizumab, which achieved sustained response to secukinumab. Clinicians should be cognizant of autoimmune-related adverse effects following immunotherapy and promptly recognize these complications. Optimal treatment and duration are in question, and further research should be conducted to assess long-term effects.

\section{Additional Information \\ Disclosures}

Human subjects: Consent was obtained by all participants in this study. Centralized Algorithms for Research Rule on IRB Exemption (C.A.R.R.I.E) issued approval 2019-181. Dear publisher, This research activity was determined to be exempt or excluded from IRB oversight in accordance with current regulation and institutional policy. Our internal reference number for this determination is 2019-181. All research materials herein, in whole or in part, are subject to copyright protection by HCA Healthcare and/or one of its subsidiaries. All rights reserved. Conflicts of interest: In compliance with the ICMJE uniform disclosure form, all authors declare the following: Payment/services info: All authors have declared that no financial support was received from any organization for the submitted work. Financial relationships: All authors have declared that they have no financial relationships at present or within the previous three years with any organizations that might have an interest in the submitted work. Other relationships: All authors have declared that there are no other relationships or activities that could appear to have influenced the submitted work.

\section{References}

1. Keytruda: PD-1 receptor blockade (n.d.). (2019). https://www.keytruda.com/hcp/mechansim-of-action/.

2. Galluzzo M, Talamonti M, Di Stefani A, Chimenti S: Linear psoriasis following the typical distribution of the 


\section{Cureus}

sciatic nerve. J Dermatol Case Rep. 2015, 9:6-11. 10.3315/jdcr.2015.1189

3. Spathas N, Economopoulou P, Cheila M, Kotsantis I, Fanouriakis A, Kassara D, Psyrri A: Inflammatory arthritis induced by pembrolizumab in a patient with head and neck squamous cell carcinoma. Front Oncol. 2018, 8:409-2018. 10.3389/fonc.2018.00409

4. Frieder J, Kivelevitch D, Menter A: Secukinumab: a review of the anti-IL-17A biologic for the treatment of psoriasis. Ther Adv Chronic Dis. 2018, 9:5-21. 10.1177/2040622317738910

5. De Bock M, Hulstaert E, Kruse V, Brochez L: Psoriasis vulgaris exacerbation during treatment with a PD-1 checkpoint inhibitor: case report and literature review. Case Rep Dermatol. 2018, 10:190-197. 10.1159/000491572 